\title{
A Simple Combined Antegrade Radiological and Retrograde Endoscopic Procedure to Recanalise Fibrotic Hypopharyngo-Oesophageal Occlusions: Technical Description and Lessons from Clinical Outcome in Three Cases*
}

\author{
Mohammed S. Miah ${ }^{1}$, Ian A. Zealley ${ }^{2}$, Afshin Alijani ${ }^{3}$, Barry McGuire ${ }^{4}$, \\ Rodney E. Mountain ${ }^{1}$, Suresh Mahendran ${ }^{1 \#}$ \\ ${ }^{1}$ Department of Otorhinolaryngology-Head \& Neck Surgery, Ninewells Hospital and \\ University of Dundee Medical School, Dundee, UK \\ ${ }^{2}$ Department of Radiology, Ninewells Hospital and University of Dundee Medical School, Dundee, UK \\ ${ }^{3}$ Department of Upper Gastrointestinal Surgery, Ninewells Hospital and University of \\ Dundee Medical School, Dundee, UK \\ ${ }^{4}$ Department of Anaesthesia, Ninewells Hospital and University of Dundee Medical School, Dundee, UK \\ Email: "sureshmahendran@nhs.net
}

Received June 2, 2013; revised June 29, 2013; accepted July 9, 2013

Copyright (C) 2013 Mohammed S. Miah et al. This is an open access article distributed under the Creative Commons Attribution License, which permits unrestricted use, distribution, and reproduction in any medium, provided the original work is properly cited.

\begin{abstract}
Background: Complete hypopharyngo-oesophageal occlusion is a rare complication of head and neck radiotherapy and a range of other conditions. Absolute dysphagia is accompanied by aspiration and dependence on gastrostomy feeding. The condition presents a substantial management challenge. Surgical approaches to re-establish pharyngo-oesophageal continuity are varied, highly invasive and are associated with unpredictable outcomes. Minimally invasive techniques employing endoscopic and radiological techniques are emerging. This report describes a multidisciplinary approach which translates two interventional radiology techniques used in the management of central venous occlusions and biliary strictures to the management of three cases of complete hypopharyngo-oesophageal occlusion. Methods: Three cases with different underlying aetiologies had treatment initiated between 2009 and 2011. Antegrade pharyngoscopic access to the occlusions was accompanied by retrograde endoscopic access via a small gastrostomy. Luminal continuity was re-established by the interventional radiology technique of "sharp recanalisation" followed by passage of a wide bore nasogastric tube which was maintained in situ for 4 - 6 months, a duration of treatment analogous to that applied in the radiological management of fibrotic biliary strictures. After treatment a radiological contrast swallows examination was performed to gauge the calibre of the re-established lumen, assess functionality and to rule out aspiration. Results: Pharyngo-oesophageal continuity was re-established in all three cases on the first attempt. No complications occurred as a result of the procedures. In two cases, the excellent swallowing function was re-established, although one of these required prolonged post-treatment adjuvant interventions. In one case no swallowing function resulted, despite apparently successful re-establishment of luminal continuity. Conclusions: Complete fibrotic occlusion of the hypopharyngo-oesophageal lumen is rare and presents a substantial management challenge. A minimally invasive treatment combining antegrade radiological and retrograde endoscopic approaches resulted in successful re-establishment of luminal continuity in three cases of complete fibrotic occlusion of the hypopharyngo-oesophageal lumen. However variable responses to treatment suggest that both the underlying aetiology and the chronicity of the occlusion may influence the likelihood of a successful functional outcome. Until definitive management guidelines are established, we suggest that such cases are managed only by motivated multidisciplinary teams keen to develop their expertise in this area.
\end{abstract}

Keywords: Hypopharynx; Upper Oesophagus; Fibrotic Occlusion; Rendezvous Technique; Pharyngo-Oesophageal Continuity

\footnotetext{
"Meetings: The work was presented at the Tri-Society of Head \& Neck Oncology Meeting on 2nd September 2011 in Singapore.

${ }^{\#}$ Corresponding author.
} 


\section{Introduction}

Complete fibrotic occlusion of the hypopharyngo-oesophageal lumen is an uncommon but important complication of successful chemoradiotherapy treatment for head and neck cancers [1]. Fibrotic occlusion may also occur after head and neck surgery, corrosive ingestion, chronic gastro-oesophageal reflux disease, neuromuscular disorders or sepsis [2,3]. Affected patients are severely debilitated. Their absolute dysphagia necessitates dependence on gastrostomy feeding but they remain at risk from saliva aspiration.

A variety of techniques to re-establish the pharyngooesophageal lumen have been described, including major surgical procedures involving excision of the occluded segment followed by reconstruction: this approach is associated with substantial surgical risk and outcomes are unpredictable [2-5]. The surgical approach may require multiple procedures followed by repeated dilatation procedures to maintain the established lumen.

We describe the successful translation of two interventional radiology techniques which have been successfully applied successfully to re-establish anatomic pharyngooesophageal continuity in patients without recourse to major surgery. We present our experience managing three patients with differing aetiologies and report outcomes.

\section{Materials and Methods}

Between March 2009 and August 2011 three patients with complete fibrotic occlusions of the hypopharynx/upper-oesophagus were referred to the otolaryngology department. All patients had complete dysphagia and were dependent on gastrostomy tube feeding.

\subsection{Case 1}

A 62-year-old male smoker developed absolute dysphagia soon after treatment of a T3NOM0 laryngeal carcinoma by chemoradiotherapy. Mucositis led to intolerance of oral feeding and he became totally dependant on his gastrostomy feeding tube. Once the mucositis had resolved it became apparent that he had developed absolute dysphagia. Total occlusion of the hypopharynx/upperoesophageal segment was observed at rigid pharyngoscopy and at barium swallow (Figure 1(A)).

\subsection{Case 2}

A 76-year-old female presented with a 10-year history of progressive dysphagia to solids and semi-solids associated with weight loss and recurrent chest infections. This was accompanied by episodes of absolute dysphagia. A previous barium swallow had demonstrated delay in initiating swallowing with marked prominence of crico- pharyngeus with proximal hold up in the hypopharynx. There was a prior history of chronic gastroesophageal reflux disease and Sjogren's syndrome. Previous flexible oesophagoscopy had identified a tight cricopharyngeal sphincter which led to rigid oesophagoscopy and dilatation, this procedure being repeated a further five times, followed by two external approach cricopharyngeal myotomy procedures and three botulinum toxin injections. These various procedures led to transient symptomatic improvement but eventually the absolute dysphagia could not be resolved and she became dependent on gastrostomy feeding. At this stage rigid pharyngoscopy and barium swallow demonstrated total fibrotic occlusion of the pharyngo-oesophageal lumen (Figure 1(B)).

\subsection{Case 3}

A 46-year-old male non-smoker presented with absolute dysphagia two years after treatment of a T3N2M0 lateral pharyngeal wall carcinoma with ablative and reconstructive surgery (split-lip mandibulotomy, excision of tumour, radial forearm free flap reconstruction and neck dissection). Post-operatively he had struggled with oral intake and this deteriorated further during chemoradiotherapy: as he developed significant mucositis he became totally dependant on his gastrostomy feeding tube. Rigid pharyngoscopy and barium swallow demonstrated total fibrotic occlusion of the pharyngo-oesophageal lumen (Figure $1(C)$ ).

\subsection{Technical Note}

The principal aim of the re-canalisation procedure we describe is to re-establish anatomic pharyngo-oesophageal continuity without recourse to major surgery. The technique we have employed to re-establish and maintain the pharyngo-oesophageal lumen involves adaptation of two interventional radiology techniques; the first developed to re-establish luminal continuity in occluded great veins in the mediastinum (the so-called "sharp recanalisation" procedure) and the second developed to manage challenging bile duct strictures non-operatively (Figure 2) [6, 7].

The procedure is performed under general anaesthetic. The initial step is to establish that the occlusion is short. If an occlusion had been greater than $3-4 \mathrm{~cm}$ in length we would not have proceeded to the "sharp recanalisation" technique as the risk of injury to non-target structures in the neck would have been considered too high. A rigid pharyngoscope is clamped into position such that that the upper limit of the occlusion is both visualised and accessible. Through an upper midline mini-laparotomy (3 cm in length) the gastric body is delivered and a $12 \mathrm{~mm}$ conventional laparoscopic port is inserted into the stomach and secured with a purse string suture. The 


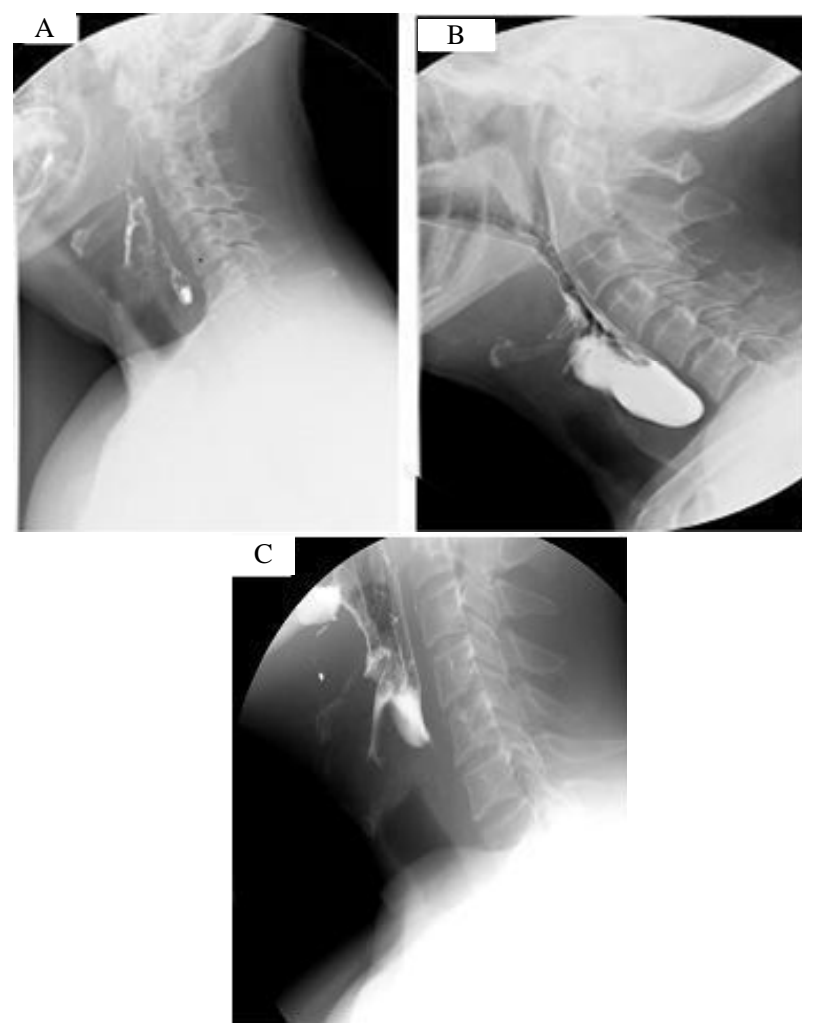

Figure 1. Pre-treatment radiological contrast swallow images for Cases 1-3, labeled A-C respectively. In each case complete occlusion of the hypopharyngo-oesophageal lumen is evident: no contrast passes into the cervical oesophagus.

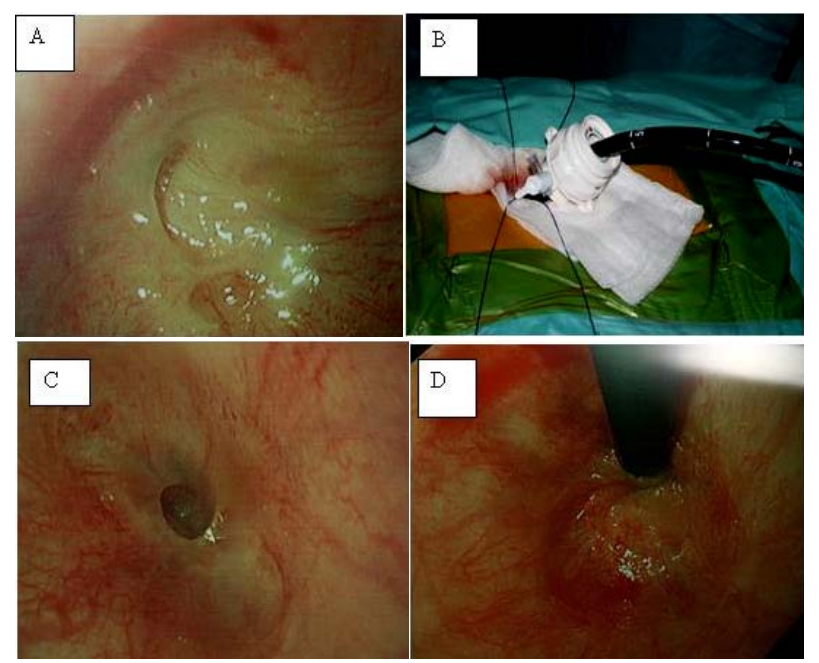

Figure 2. Images A-D demonstrate the key elements of the recanalisation procedure. A: Rigid pharyngoscopic view of the upper limit of the occlusion; B: Retrograde flexible oesophagoscopy via mini-laparotomy and gastrostomy; C: Flexible oesophagoscopic view of the lower limit of the occlusion; D: Endoscopic view of puncture through the occlusion using the radiological needle.

laparoscopic port thus creates a convenient air-tight retrograde access into the stomach for the insertion of the flexible oesophagoscope. The port also facilitates gastric insufflation with carbon dioxide, in addition to the endoscopic gas insufflation, for better visualization and negotiation of the gastro-oesophageal junction. The flexible oesophagoscope is advanced up the oesophagus until the lower aspect of the occlusion is encountered. Once the antegrade pharyngoscope and the retrograde flexible oesophagoscope are in place an image intensifier is positioned for lateral fluoroscopy of the cervical region. Fluoroscopy is employed to gauge the length of the occluded segment. An interventional radiologist then uses a long needle and co-axial catheter assembly (Rosch-Uchida Transjugular Liver Access Set, Cook Medical, Amersham, UK) to puncture across the occlusion from the upper end, using a combination of direct visualization and fluoroscopy for needle guidance. Entry into the distal oesophageal lumen is confirmed by watching the flexible oesophagoscope images from below the occlusion. After the co-axial needle and catheter assembly has been advanced into the oesophageal lumen below the occlusion the needle component is withdrawn and a $260 \mathrm{~cm}$ stiff radiological guidewire (Amplatz, Boston Scientific, Natick, MA, USA) passed down the catheter into the distal oesophageal lumen where it is grasped via the flexible oesophagoscope, thus achieving "rendezvous" between the patent lumen above and below the occlusion. The guidewire is then withdrawn down through the distal oesophagus and out through the mini-laparotomy, thereby creating through-and-through guidewire access across the occlusion.

Gentle dilatation of the occlusion is performed using an $8 \mathrm{~mm}$ balloon catheter in order to create a channel wide enough to accommodate passage of an 18 Fg Ryle's tube. In order to convert the per-oral guidewire access across the occlusion into per-nasal access a radiological catheter is inserted through the nose and its tip pulled out of the mouth. The upper end of the guidewire is then fed retrogradely into the tip of the per-nasal catheter and, once a sufficient length of guidewire has been thus deployed, the per-nasal catheter containing the upper end of the guidewire is withdrawn back through the mouth and nose until the guidewire is exiting per nares.

At this stage a modified 18 Fg Ryle's tube is passed over the guidewire and through the occlusion, under fluoroscopic guidance. The Ryle's tube is modified by cutting a cruciate incision in its tip in order that it may be advanced over the guidewire with the smallest possible profile: this manoeuvre avoids the larger, eccentric profile which would result if the Ryle's tube were advanced over the guidewire via the side-hole at the tip of the tube. Finally the Ryle's tube is secured using a nasal septal bridle and the gastrostomy and mini-laparotomy are closed.

Postoperatively, the patients receive a seven day course 
of proton pump inhibitor to minimize the risk of bleed from the gastrostomy site. A post-operative chest radiograph was performed to rule out a pneumomediastinum, and the patients were observed closely in order to identify mediastinitis early should it develop. In all three cases the Ryle's tubes were left in-situ for a minimum of 4 - 6 months. This duration of dwell time was based on long-term patency reported in analogous studies on the percutaneous catheter management of fibrotic bile duct strictures [7].

The patients were encouraged to swallow fluids as and when they feel able, and were followed-up as outpatients initially at two weeks and thereafter at two monthly intervals to gauge tolerance of the treatment regime and assess progress on swallowing. After 4 - 6 months rigid pharyngoscopy under general anaesthetic was performed to inspect and record the condition of the re-established lumen, and the Ryle's tubes were removed. Subsequently a radiological water-soluble contrast swallow examination was performed to assess the patency and function of the previously occluded segment.

\section{Results}

Re-establishment of pharyngo-oesophageal luminal patency was achieved using this technique was successful on the first attempt in all three cases. A functional swallow was re-established in two of the three cases. No complications were observed.

\subsection{Case 1}

During the recanalisation procedure the occluded segment was found to be $1.5 \mathrm{~cm}$ long. The patient reported some discomfort from the presence of the Ryle's tube but tolerated its presence for the recommended duration. During the later weeks of the treatment period the patient reported symptoms consistent with bile reflux. At rigid pharyngoscopy at the end of treatment a patent lumen substantially wider than the Ryle's tube itself was evident around the tube: there was also evidence of bile reflux (Figure 3). The patient tolerated oral fluids and soft diet soon after removal of the Ryle's tube. The subsequent radiological water-soluble contrast swallow study demonstrated re-establishment of the hypopharynx/upper-oesophagus lumen with no hold up of contrast (Figure 4(A)). The previously occluded segment was found to be distensible and no aspiration was observed. The gastrostomy tube was removed two weeks after removal of the Ryle's tube. At 42 month follow-up the patient was managing an oral diet, including most solids and all fluids, and had gained 13 kilograms in weight. His emotional affect, previously withdrawn and depressed, was enormously improved. No further intervention has been required.

\subsection{Case 2}

During the recanalisation procedure the occluded segment was found to be $2 \mathrm{~cm}$ long. The patient tolerated the presence of the Ryle's tube very well. However, despite the fact that hypopharyngeal-oesophageal luminal continuity was achieved using the technique described the patient was unable to swallow fluids during the whole of the treatment period, and also after removal of the Ryle's tube at 6 months. A radiological water-soluble contrast study demonstrated a rat's tail appearance of the cervical oesophagus at the level of the previously recanalised occlusion, accompanied by dilatation of the oesophagus above this level and hold up of contrast (Figure 4(B)). No active peristalsis was observed. To date, this patient has not been able to produce a functional swallow.

\subsection{Case 3}

During the recanalisation procedure the occluded segment was found to be $2 \mathrm{~cm}$ long. At rigid pharyngoscopy the fibrotic occlusion appeared to be substantially more dystrophic and distorted than the other two cases. This patient did not tolerate the presence of the Ryle's tube well. An early re-inspection of the occlusion site was performed at three months at the patient's request. The re-established lumen was found to be patent and expansile clinically: the lumen accommodated the 18 Fg Ryle's tube alongside two fully inflated $8 \mathrm{~mm}$ balloon catheters (Figure 5). Biopsies were taken and these demonstrated the presence of active granulation tissue. In view of this finding the patient agreed to persist with the Ryle's tube in situ for a further month. During this period intensive swallow therapy support was also instituted. After a total of four months of treatment the patient was still unable to swallow fluids but reported reflux of gastrostomy feed solution. The Ryle's tube was removed and the patient

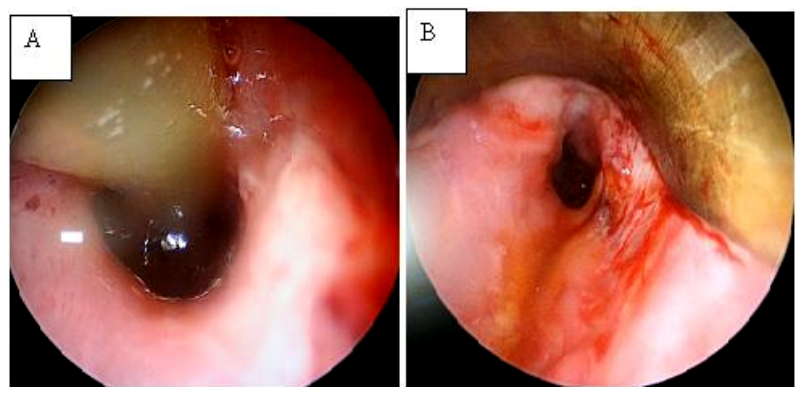

Figure 3. Rigid pharyngoscopic images of the mature recanalised hypopharyngo-oesophageal lumen in case 1 before (image A) and after (image B) removal of the Ryle's tube which had been in situ for six months. The diameter of the lumen was substantially greater than the Ryles' tube itself. Bile reflux was evident after removal of the Ryle's tube (image B): this patient had reported symptoms of bile reflux during the latter weeks of treatment. 
continued with swallowing therapy exercises. Over the next two months further balloon dilatation procedures were performed under rigid pharyngoscope endoscopic guidance in order to maintain the calibre of the recanalised lumen during this extended period of swallowing therapy exercises. Six months after the recanalisation procedure the patient was swallowing fluids with ease, and has subsequently been building up consistencies. A radiological water-soluble contrast study at nine months post re-canalisation demonstrated a patent and functional pharyngo-oesophageal lumen (Figure 4(C)). At 18 months follow-up, this patient is now managing soft diet and semi-solids.

\section{Discussion}

Management of fibrotic hypopharyngo-oesophageal occlusions presents a major challenge. Surgical resection of the occluded segment and reconstruction carries substantial procedural risks and outcomes are unpredictable [2, 3,8].

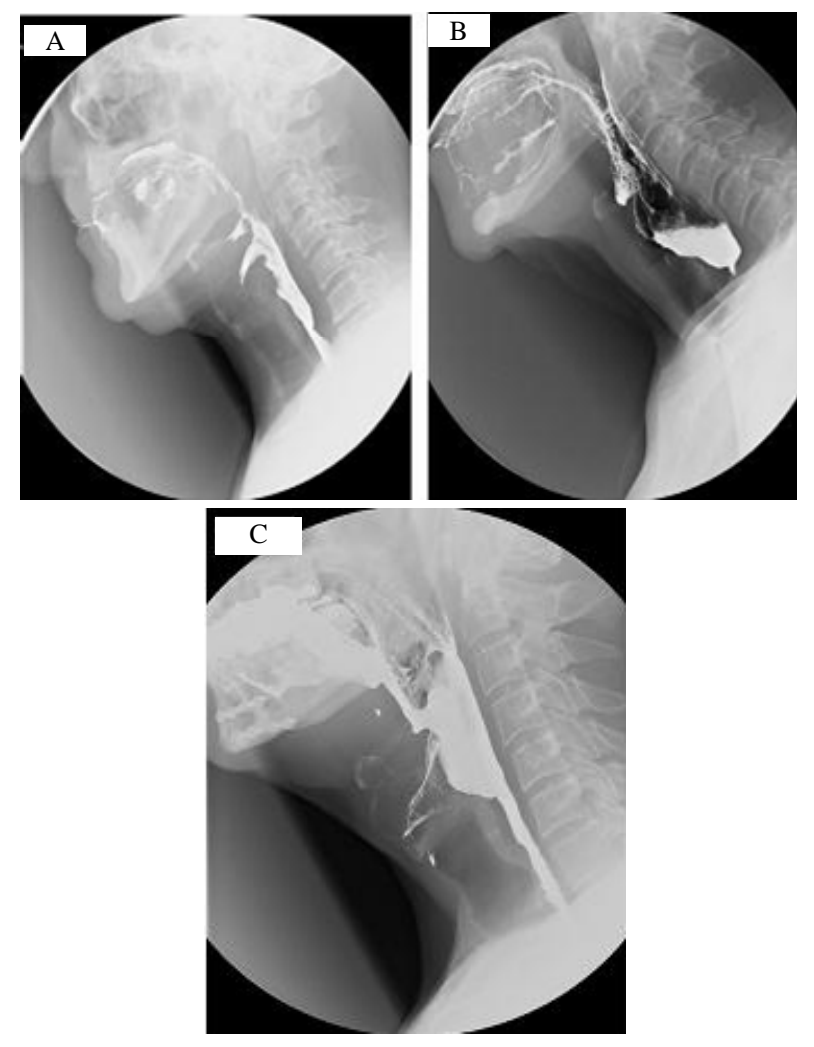

Figure 4. Post-treatment radiological contrast swallow images for Cases 1-3, labeled A-C respectively. Images $A$ and $C$ demonstrate the functional recanalised hypopharyngooesophageal lumen which was the final result for cases 1 and 3 respectively. Image $B$ demonstrates the non-functional outcome which was the final outcome for case 2 . There is a rat's tail appearance in the cervical oesophagus with no evidence of peristaltic activity, and hold up of contrast in the dilated hypopharynx above.
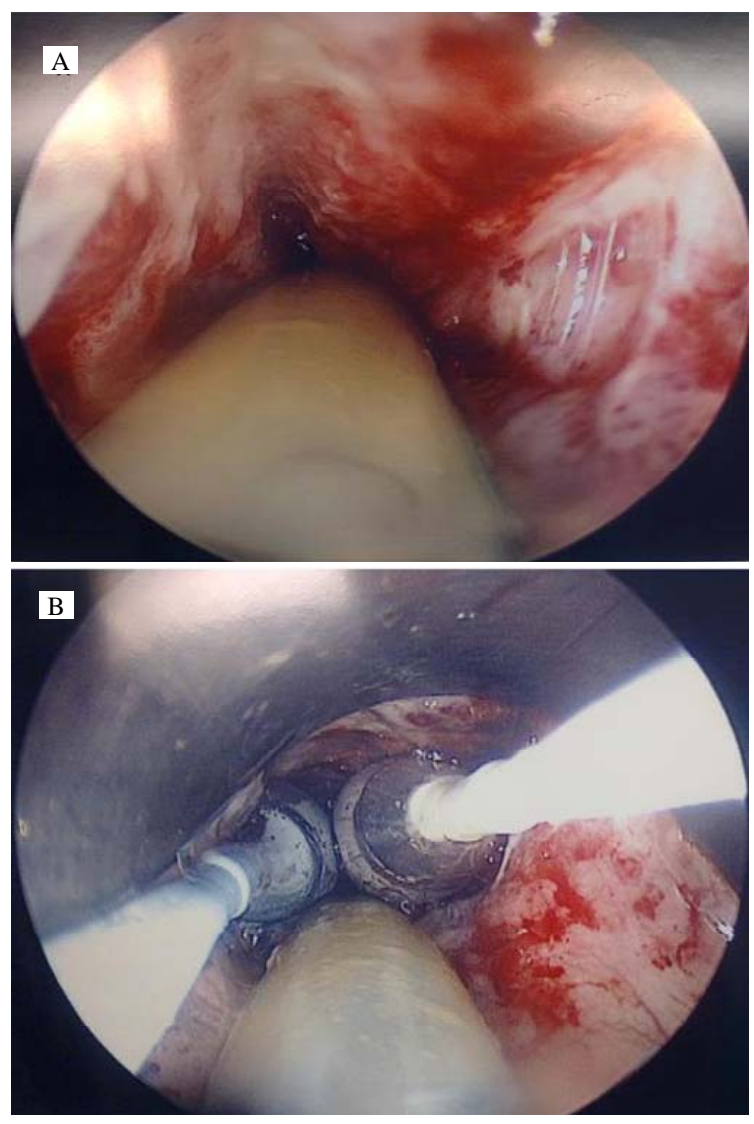

Figure 5. Rigid pharyngoscope images in Case 3 before balloon dilatation (A) and during dilation of twin radiological balloon catheters (B). The images demonstrate that the recanalised lumen exhibits elasticity and that it can accommodate the $18 \mathrm{Fg}$ Ryle's tube and two inflated $8 \mathrm{~mm}$ balloon catheters simultaneously. Despite this apparently excellent outcome this patient required extensive post-treatment swallowing therapy and several further one-off dilatation procedures before a satisfactory functional swallow was re-established.

Interventional radiology "sharp recanalisation" techniques applied from the upper end of the occlusion, without simultaneous access at the lower aspect of the occlusion, may be unsafe because of the potential for inadvertent puncture of non-target structures and creation of fistulae to the pleura, lung, mediastinum, or airway $[4,5]$.

The technique described is an adaptation of percutaneous, radiologically-guided approaches to the management of occlusions of the great veins of the mediastinum and bile duct strictures (Figure 2) [6,7]. Success rates for recanalization of mediastinal great veins ranging between $80 \%$ - 100\% [6] have been reported. The long term success employing the technique with bile duct strictures is dependent on the duration the stent is in situ with minimum recommended duration of 6 months [7]. Further support for this has been gained from our own experience managing patients with severe hypopharynx/upper-oesophagus strictures with radiological balloon dilatation 
followed by long-term Ryle's tube placement (several months). We have previously reported durable functional outcomes achieved with 1 - 4 years follow-up in three patients [9].

Concurrent antegrade-retrograde rendezvous technique has been described previously, with different technical approaches and variable outcomes [4,5,8,10,11]. Most commonly the rendezvous technique relies on retrograde flexible oesophagoscopy or fluoroscopy through the gastrostomy tube site. During an early attempt in our experience using this approach we attempted this manoeuvre but were unable to cannulate the oesophagus. This may have been due to our inexperience and/or the involutional changes in the oesophagus and at the gastrooesophageal junction which occur after the prolonged oesophageal redundancy which inevitably results from the upstream occlusion. We had also learned of an attempted retrograde oesophagoscopy via the gastrostomy site at another centre which resulted in loss of continuity at the gastrostomy site, requiring immediate laparotomy for repair and creation of a second remote gastrostomy. It was these experiences which led us to opt for securing robust, controllable retrograde access by means of a mini-laparotomy. This limited open surgical approach was selected in preference to a laparoscopic approach in view of the presence of local adhesions related to the pre-existing gastrostomy feeding tube insertion. A further difference between the technique described and previously reported rendezvous procedures is the long duration (4 - 6 months) of nasogastric tube placement which follows the initial recanalisation procedure.

Oxford et al. and Maple et al. described the short term use of nasogastric tubes as stents to maintain patency of the re-established hypopharyngo-oesophageal lumen [8, 10] after serial balloon dilatations. In their series' gastrostomy tube feeding was discontinued in $67 \%$ and $57 \%$ respectively. Five of the six patients treated by Oxford et al maintained hypopharyngo-oesophageal luminal patency with a minimum follow-up of 12 months. Two of the seven cases described by Maple et al developed evidence of oesophageal microperforation after their rendezvous procedures, and a further case developed early re-stenosis requiring repeated subsequent dilatation procedures. A paediatric case described by Zur et al. required seventeen dilatation procedures during an 18month period, accompanied by kenalog injections and topical mitomycin-C application on two occasions [11]. An encouraging observation made at rigid pharyngoscopy at the end of the six month treatment period in the first case described here was the large calibre of the reestablished hypopharyngo-oesophageal lumen in comparison to the diameter of the Ryle's tube (Figure 3). We hypothesise that this phenomenon may have been due to continuous relative movement of the tube and the muco- sal surface during the treatment period.

\subsection{Anatomic versus Functional Outcome}

The procedure described successfully re-established pharyngo-oesophageal luminal continuity in all three cases on the first attempt. However the varied outcomes indicate that re-establishment of luminal continuity does not necessarily confer the immediate return of a functional swallow.

Two of the cases had fibrotic occlusion after oncological treatment for pharyngeal cancers: Case 1 had chemoradiotherapy only whereas Case 3 had major ablative and reconstructive surgery followed by radiotherapy. At the time of recanalisation it was noted that Case 3 appeared to have a greater extent of mature fibrosis than the other cases. We speculate that this is due to two factors 1 ) the presence of a free flap and 2) the prolonged interval to the recanalisation attempt.

Of all 3 cases, Case 1 has clearly enjoyed the best outcome developing a durable functional swallow immediately after treatment. Case 3 required intensive swallow therapy and repeated dilatations before also developing a durable functional swallow: his own motivation and perseverance have undoubtedly been an important contributing factor in this outcome.

Case 2 never established a functional swallow. While the precise aetiology of the occlusion in this case was unclear the history supported a diagnosis of chronic gastroesophageal reflux disease leading to stricture formation and eventually complete fibrotic occlusion. The multiple prior interventions (five dilatations, two myotomies and three botulinum toxin injections) may have exacerbated the neurological component of the chronic inflammatory process at the site of occlusion. Following apparently successful treatment a radiological contrast swallow demonstrated complete absence of any muscular contractility around the site of the recanalised occlusion. It appeared that there was no effective propulsive force to propel ingested fluid through the abundant, redundant hypopharyngeal mucosa above the recanalised segment. An attempt to place a stent across the recanalised segment was unsuccessful; a failure attributed to the presence of the excess mucosal tissue. No further intervention is planned. This patient remains dependent on gastrostomy tube feeding.

The outcome in case 2 suggests that re-establishment of functional swallow is more likely to be successful in patients with no prior history of oesophageal dysfunction before developing fibrotic occlusion. The outcome in case 3 suggests that a combination of ablative and reconstructive surgery with adjuvant treatments, followed by a prolonged period of absolute dysphagia prior to the intervention described, may have all contributed towards a more protracted course to a functional outcome. The ex- 
perience of the three cases suggests that some degree of patient selection coupled with careful patient counseling may be important if recanalisation procedures such as that described are to be successful in re-establishing a functional swallow. The scarcity of cases of complete occlusion is reflected in the limited literature on minimally invasive recanalisation techniques such as that described. In view of this, and the varied outcomes described in this manuscript, it is suggested that until definitive advice on patient selection and recanalisation techniques has been developed these cases are managed only in centres where efforts are being made to develop an experience base. It is also suggested that a multidisciplinary team approach, with detailed prospective planning and patient education, is necessary for minimally invasive recanalisation therapy techniques such as this to be effective.

\subsection{Ethical Considerations}

Thankfully despite the increasing use of chemoradiotherapy as primary treatment modality in locally advanced malignancies of the upper aerodigestive system especially those with Human Papilloma Virus associated aetiology, cases of complete fibrotic occlusion are rare. Although the technique described is novel it is, in effect, merely an adaptation of existing surgical and, interventional radiology procedures for which complications and outcomes are established. This background of a translational approach to therapy formed the basis of our discussion with all three patients. However they were also informed that the combination of techniques was novel and that consequently the outcomes pertaining to swallow could not be predicted with any degree of certainty. All three patients benefited from a multidisciplinary team approach and were fully cognisant of the fact that the experience base for minimally invasive recanalisation procedures such as that described is small and in evolution.

\section{Conclusion}

Total fibrotic occlusion of the hypopharynx/upper-oesophagus tract is rare but poses a substantial management challenge. The minimally invasive recanalisation described combines elements of existing surgical and interventional radiology techniques, none of which require extraordinary or unusual experience or expertise. Although luminal continuity was re-established in all three cases, the variable outcomes suggest that the underlying aetiology and the chronicity of the occlusion may be important variables influencing the likelihood of re-establishing a functional swallow. We suggest that until definitive management algorithms can be developed, this type of procedure only be performed for patients who have been appropriately counseled and only by motivated multidisciplinary teams who are keen to develop their expertise in this area.

\section{REFERENCES}

[1] H. B. Caglar, R. B. Tishler, M. Othus, et al., "Dose to Larynx Predicts for Swallowing Complications after Intensity-Modulated Radiotherapy,” International Journal of Radiation Oncology Biology Physics, Vol. 72, No. 4, 2008, pp. 1110-1118. doi:10.1016/j.ijrobp.2008.02.048

[2] M. Chirica, C. de Chaisemartin, N. Munoz-Bongrand, et al., "Colonic Interposition for Esophageal Replacement after Caustic Ingestion,” Journal De Chirurgie (Paris), Vol. 146, No. 3, 2009, pp. 240-249.

[3] J. W. Hong, H. S. Jeong, D. H. Lew, et al., "Hypopharyngeal Reconstruction Using Remnant Narrow Pharyngeal Wall as Omega-Shaped Radial Forearm Free Flap,” Journal of Craniofacial Surgery, Vol. 20, No. 5, 2009, pp. 1334-1340.

[4] S. J. Tang, S. Singh and J. M. Truelson, “Endotherapy for Severe and Complete Pharyngo-Esophageal Post-Radiation Stenosis Using Wires, Balloons and Pharyngo-Esophageal Puncture (PEP) (with Videos)," Surgical Endoscopy, Vol. 24, No. 1, 2010, pp. 210-214.

[5] B. Pogorzelski, R. Kiesslich and W. Mann, ”Rendezvous Technique for Complete Hypopharyngeal Stenosis. Indications and Variations of Combined Endoscopy," HNO, Vol. 57, No. 8, 2009, pp. 781-788.

[6] S. Athreya, P. Scott, G. Annamalai, et al., "Sharp Recanalization of Central Venous Occlusions: A Useful Technique for Haemodialysis Line Insertion,” British Journal of Radiology, Vol. 82, No. 974, 2009, pp. 105108. doi:10.1259/bjr/19820366

[7] S. Misra, G. B. Melton, J. F. Geschwind, et al., "Percutaneous Management of Bile Duct Strictures and Injuries Associated with Laparoscopic Cholecystectomy: A Decade of Experience," Journal of the American College of Surgeons, Vol. 198, No. 2, 2004.

doi:10.1016/j.jamcollsurg.2003.09.020

[8] J. T. Maple, B. T. Petersen, T. H. Barron, et al., "Endoscopic Management of Radiation-Induced Complete Upper Esophageal Obstruction with an Antegrade-Retrograde Rendezvous Technique,” Gastrointestinal Endoscopy, Vol. 64, No. 5, 2006, pp. 822-828.

doi:10.1016/j.gie.2006.06.026

[9] M. S. Miah, I. A. Zealley, R. E. Mountain and S. Mahendran, "Severe Benign Hypopharynx/Upper-Oesophageal Strictures: Successful Treatment with a Novel Interventional Radiology Technique in Three Patients,” Clinical Otolaryngology, Vol. 37, No. 4, 2012, pp. 313-317.

[10] L. E. Oxford and Y. Ducic, "Retrograde Balloon Dilation of Complete Cervical Esophageal and Hypopharyngeal Strictures,” The Journal of Ototaryngotography, Vol. 55, No. 5, 2006, pp. 327-331. doi:10.2310/7070.2005.0127

[11] K. B. Zur, P. E. Putnam and M. J. Rutter, "Combined Retrograde and Anterograde Hypopharyngeal Puncture and Dilatation in a Child with Complete Hypopharyngeal Stenosis," International Journal of Pediatric Otorhinolaryngology, Vol. 71, No. 1, 2007, pp. 153-157. doi:10.1016/j.ijporl.2006.07.023 